Ordinal positions of letters within words as concepts: Following the studyre

\title{
Effect of assigning identical responses to words sharing letters in various ordinal positions ${ }^{1}$
}

\author{
DOUGLAS L. NELSON, STEVEN \\ BERCOV, and LAUREN LESLIE, \\ University of South Florida, Tampa, Fla. \\ 33620
}

Eight stimulus words were constructed by using all combinations of two different first, middle, and last letters. Number of single digit responses were varied 12,4 vs 8). Within the 2- and 4-response conditions, locus of response assignment was manipulated so that words sharing letters within particular ordinal positions also shared responses. Each $S$ learned a single list. On the basis of data suggesting that initial, final, and then medial ordinal positions represent, in this order, the most critical determinants of word processing, it was predicted that ease of acquisition for the locus of response assignment conditions would also reflect this ordering. However, acquisition was easiest when identical responses were assigned to words having identical first letters, next with middle-letter assignment, and most difficult with third-letter assignment.

Recent evidence suggests that initial and final letters arc more influential determinants of word processing than are medial letters, and initial are more critical than final letters (e.g., Bruner \& O'Dowd, 1958; Carson, 1961; Horowitz, White, \& Atwood, 1968). This differential sensitivity to ordinal positions of letters within words may account for findings obtained in studies in which there were variations in the positions of letters shared by the stimuli (i.e., jdentical letters located in the first, second, etc., positions, reading the the trigram from left to right). When discriminations between stimuli were required as in paired-associate (Nelson \& Rowe, 1969) and serial-recall learning (Nelson, 1969), difficulty of acquisition was greatest when the identity was located within initial positions, next in terminal positions, and least within medial positions. Orders of difficulty by location condition were reversed when shared letter stimuli could be grouped or recalled together as in free-recall learning (Nelson, 1969) and when the letter sharing was between a paired-associate stimulus and its response (Nelson \& Garland, in press).

The purpose of this investigation was to test the limitations of the ordinal-position effect within the context of a modified concept-formation task. A single eight-item stimulus list was constructed, using all combinations of two different first, middle, and last letters. Number of possible single-digit responses $(2,4$, or 8$)$ and ordinal position of response assignment (first, middle, etc.) were varied. Within the two- and four-response conditions, location of response assignment was varied by assigning identical responses to stimuli sharing letters within given ordinal positions. For example, with first-letter position assignment, all words beginning with $B$ had the same response and all those beginning with $\mathrm{L}$ shared the other response. In this case, the concept was "words with identical first letters have the same response." In accord with previous data, it was hypothesized that acquisition in the two-response conditions should be most difficult when identical responses were assigned to identical middle (M) letters, next with last- (L) letter assignment, and least with first- (F) position assignment. Within the four-response conditions, in which identical responses were assigned to identical bigrams, acquisition ease should increase in the order of middle-and-last $(M+L)$, first-and-middle $(F+M)$, and first-and-last $(F+L)$. In addition, as the number of possible responses increases, acquisition ease should decrease (e.g., Richardson, 1958).

\section{EXPERIMENT 1 \\ Verbal Material}

Each $S$ acquired one of seven eight-item paired-associate (PA) lists. The stimuli were three-letter words constructed by using all combinations of two different first, middle, and last letters: BAD, BAG, BED, $B E G$, LAD, LAG, LED, LEG. In the two-response conditions, the digits 1 and 8 were assigned randomly to stimuli, with the restriction that stimuli with identical $F$, $\mathrm{M}$, or L letters share the same responses. In the four-response conditions, the single-digit responses $1,5,6$, and 8 were assigned randomly to stimuli, with the restriction that items with identical $F+M$, or identical $\mathrm{F}+\mathrm{L}$, or identical $\mathrm{M}+\mathrm{L}$ letters had the same responses. In the eight-response condition, the digits 1-8 were assigned randomly to stimuli so that each response was assigned to a single stimulus.
Following the study trial, each PA list was practiced in an individual session by the anticipation method for a total of 25 trials. On the initial presentation of the list and on every trial thereafter, $S$ was required to pronounce each stimulus aloud on test trials and guess at its response. In the instructions, Ss were informed of the specific response set and were told that each response was paired with a single word (eight-response condition), with two different words (four-response conditions), or with four different words (two-response conditions). Data from the initial study-guess trial were omitted from statistical analysis. Slides of each stimulus and stimulus-response pair were automatically projected by a Kodak Carousel slide projector, driven by an auxiliary timer at a $2: 2-\mathrm{sec}$ rate. There was no intertrial interval. Sequences of pairs were presented in five unsystematically varying orders, with the restriction that no two successive lists begin or end with the same pair. The sequence of each of the five orders was changed for each $S$ in each condition. All Ss learned a practice list of two pairs (names-verbs) before undertaking the experimental task.

\section{Subjects}

There were seven groups of $16 \mathrm{Ss}$ drawn from an introductory psychology course who received course credit for participation. They were assigned to conditions in order of appearance in blocks of seven, with one $S$ from each condition per block. Order of conditions within blocks was determined by a table of random numbers. Each of two Es ran half the Ss in each condition.

\section{Results}

A mixed-model ANOVA (Myers, 1966) of correct responses showed that conditions, trials, and the Conditions by Trials interaction were significant, with the sources involving the repeated measures variable remaining significant following the Greenhouse \& Geisser (1959) correction. Mean correct responses increased in the order L (174.69), $\mathrm{M}(178.25)$, and $F(189.56)$ for the two-response conditions and in the order $M+L(115.12)$, $F+L(144.75)$, and F + M (148.37) for the four-response conditions. Mean correct responses for the eight-response control were 112.37. Fisher's least significant difference was 2.69 , with the $5 \%$ level as the criterion of significance, indicating that each mean was statistically different from each other mean. The significant interaction reflected the disproportionately faster acquisition rates for the two-relative to the four- and eight-response conditions.

$A$ within-S analysis of the confusion errors was performed on the data in the 
eight-responsc control condition. For each word in the list, there were seven possible types of confusion error, i.e., a response given to a particular stimulus that belongs to another stimulus in the list that shared zero, first, or middle, etc., letters with that particular stimulus. Of all errors, $94.3 \%$ could be classified on this basis, the remaining $5.7 \%$ representing omission errors. ANOVA of these errors revealed a significant confusion error effect. The subsequent least significant difference value was 2.77 . Confusion errors increased in the order of zero (7.68), M (7.81), L (9.31), and $F(11.50)$. For stimuli sharing two letters, the order was $M+L(9.25), \quad F+M(16.31), \quad$ and $F+L(17.44)$. The patterns of differences were consistent with those found in between-S variations of locus (Nelson \& Rowe, 1969).

\section{EXPERIMENT 2}

Since the stimuli of Experiment 1 were CVCs, consonant-vowel pattern was confounded with locus of response assignment, i.e., for Conditions $\mathrm{F}+\mathrm{M}$, $F+L$, and $M+L$, the respective critical bigram patterns were $\mathrm{CV}, \mathrm{CC}$, and VC. If associating a response to a $\mathrm{CV}$ pattern was easier than associating a response to a $\mathrm{CC}$ pattern, then the superiority of the $F+M$ relative to the $F+L$ condition might be explained on this basis. Experiment 2 was designed to test this possibility.

\section{Verbal Material}

Generation of a stimulus set conforming to $\mathrm{CVV}$ or VCC patterns using two different first, middle, or last letters was found to be impossible. Such a set would have been ideal since, using the same words, the critical bigrams would have been of the same pattern type for the $F+M$ and $F+L$ conditions. Instead, two different lists were constructed representing the stimuli for these conditions which were, respectively, ARC, ARM, AND, ANY, OFF, OFT, OWL, OWE, and ACT, ANT, ARK, ASK, ODD, OLD, OWL, OIL. All stimuli conformed to the VCC pattern except the last stimulus in each list. Every other pair of these stimuli shared the same randomly assigned response, $1,5,6$, or 8 . Three other stimulus lists were constructed, using bigrams drawn from the stimulus list of
Experiment 1. To form the stimuli for the bigram control condition for $F+M$, the last letters of the CVC trigrams were deleted, resulting in $\mathrm{CV}$ bigrams. Similarly, for Conditions $\mathrm{F}+\mathrm{L}$ and $\mathrm{M}+\mathrm{L}$, respectively, the middle and final letters were deleted so that these bigrams conformed to $\mathrm{CC}$ and $\mathrm{VC}$ patterns. In the bigram conditions, each bigram appeared twice within a given trial. Procedure

The procedure, the Es, the number of Ss, and $S$ assignment were identical to Experiment 1 except that practice on the bigram conditions was continued to a maximum of 10 trials or until three consecutive trials without error.

\section{Results}

Means of correct responses for the $\mathrm{F}+\mathrm{L}$ and $\mathrm{F}+\mathrm{M}$ conditions were 155.12 and 172.56, respectively. A mixed-model ANOVA indicated that this difference and the trials effect were significant. Means of correct responses in 10 trials for the $\mathrm{CV}$, $\mathrm{CC}$, and VC conditions were, respectively, $68.81,65.87$, and 69.56. ANOVA indicated that only the trials source was significant, the $F$ for bigram conditions being less than unity.

\section{DISCUSSION}

In addition to replicating Richardson's (1958) finding that acquisition ease decreases as the number of responses increases, these results indicate that different ordinal positions within words are differentially effective as concepts. Acquisition was fastest when identical responses were assigned to identical initial letters, next for middle-letter assignment, and slowest for terminal-letter assignment. The results of Experiment 2 suggest that this ordering was a result of differential rates of concept solving and not a by-product of the confounding of consonant-vowel patterning with locus of the critical letters.

The relative ordering of conditions indicates that the words in this task were processed in a left-right rather than in a beginningend-middle sequence as has been typically found. The reason for this difference may lie in the particular characteristics of the concept-formation aspect of this task. The information that each response was paired with more than one stimulus may have invoked attempts to identify a rule that would relate stimulus letters and responses. For example, in systematically using a left-right rule, an initial attempt is made to associate responses to first letters. If this rule fails, then association to middle letters are tried, followed by attempts to associate to last letters and then to bigrams, starting from the first two letters, etc. The principal assumption of this interpretation is that, in this task, words were being analyzed into their constituent letters rather than being encoded as units. This analysis would suggest that if the concept-formation aspect of the task was eliminated, the more typical beginning-end-middle pattern of results would emerge. The pattern of confusion errors obtained in the eight-response condition of this study and other paired-associate data (Nelson \& Rowe, 1969) are consistent with this interpretation. Thus, the results of these experiments suggest that the beginningend-middle sequence effect is limited to situations in which the stimuli are processed and encoded as units.

\section{REIERENCES}

BRUNER, J. S., \& O'DOWD, D. A note on the informativeness of parts of words. Language \& Speech, 1958, 1, 98-101.

CARSON, D. H. Letter constraints within words in printed English. Kybemetic, 1961, 1, 46-54.

GREENHOUSE, S. W., \& GEISSER, S. On methods in the analysis of profile data. Psychometrika, 1959, 24, 95-112.

HOROWITZ, L. M., WHITE, M. A., \& ATWOOD, D. W. Word fragments as aids to recall: The organization of a word. Journal of Experimental Psychology, 1968, 76, 219-226.

MYERS, J. L. Fundamentals of experimental design. Boston: Allyn \& Bacon, 1966.

NELSON, D. L. Information theory and stimulus encoding in free and serial recall: Ordinal position of formal similarity. Journal of Experimental Psychology, 1969, 80, 537-541.

NELSON, D. L., \& GARLAND, R. M. Amount and locus of stimulus-response overlap in paired-associate acquisition. Joumal of Experimental Psychology, in press.

NELSON, D. L., \& ROWE, F. A. Information theory and stimulus encoding in paired-associate acquisition: Ordinal position of formal similarity. Joumal of Experimental Psychology, 1969, 79, 342-346.

RICHARDSON, J. The relationship of stimulus identity and number of responses. Journal of Experimental Psychology, 1958, 56, 478-484. NOTE

1. This research was supported by the University of South Florida Research Council and by a grant (MH 16360-01) to the first author from the National Institute of Mental Health. 